\title{
Interaction between widening of diameter of abdominal aorta and cardiovascular risk factors and atherosclerosis burden
}

\author{
Frédéric Glauser $\cdot$ Lucia Mazzolai • \\ Roger Darioli • Michèle Depairon
}

Received: 7 December 2012/ Accepted: 26 March 2013/Published online: 9 April 2013

(C) SIMI 2013

\begin{abstract}
The aim of this study was to investigate influence of traditional cardiovascular risk factors (CVRF) and subclinical atherosclerosis (ATS) burden on early stages of abdominal aortic diameter (AAD) widening among adults. 2,052 consecutive patients (P) (39\% women), mean age $52 \pm 13$ years, were prospectively screened for CVRF, ATS, and AAD. B-mode ultrasound was used to evaluate the largest $\mathrm{AAD}$ and to detect carotid and femoral atherosclerotic plaques. Mean AAD was $15.2 \pm 2.8 \mathrm{~mm}$. Atherosclerotic plaques were detected in $71 \%$ of patients. Significant univariate correlation between AAD, traditional CVRF, and ABS was found. However, multiple regression analysis showed that only seven of them were significantly and weakly correlated with $\mathrm{AAD}\left(R^{2}=0.27, p<0.001\right)$. On the other hand, a multivariate logistic analysis was used to evaluate CVRF impact on enlarged $A A D \geq 25 \mathrm{~mm}$ (EAAD) as compared to those with AAD $<25 \mathrm{~mm}$. These factors did not account for more than $30 \%$ of interaction $\left(R^{2}=0.30, p=0.001\right)$. Furthermore, despite a large proportion of patients with high number of CVRF, and subclinical ATS, rate of patients with AAD $\geq 25 \mathrm{~mm}$ was low (1\%) and scattered regardless their CHD risk score or ATS burden. In conclusion, these results suggest that although some traditional CVRF and presence of ATS are associated with early stages of EAAD, other determinants still need to be identified for a better understanding of abdominal aortic aneurysm pathogenesis.
\end{abstract}

F. Glauser $(\varangle) \cdot$ L. Mazzolai · M. Depairon

Service d'Angiologie, Lausanne University Hospital, CHUV, rue du Bugnon 21, 1011 Lausanne, Switzerland e-mail: Frederic.Glauser@chuv.ch

R. Darioli

Lausanne University, 5 chemin des Fleurs,

1007 Lausanne, Switzerland
Keywords Atherosclerosis - Aorta - Cardiovascular risk factors

\section{Introduction}

Relative role of contributors to abdominal aortic aneurysm (AAA) development is still a matter of debate. Atherosclerosis (ATS) and AAA share common risk factors, such as male sex, advanced age, and smoking habits [1]. In contrast, robust data indicate that diabetes, a major cardiovascular risk factor (CVRF), is inversely correlated with presence and progression of AAA [1, 2]. Contribution of hypertension [2,3], obesity $[4,5]$, and hypercholesterolemia $[6,7]$ in AAA development remains at present unclear. ATS and AAA differ also with respect to histological findings. Media weakening involving chronic inflammation, and extensive protease-mediated degradation, is a main feature of AAA [8, 9]. Populationbased studies have shown that the presence of peripheral vascular disease, or coronary heart disease (CHD), two well-known atherosclerosis-linked diseases, are strong predisposing factors for AAA development [10, 11]. Numerous epidemiological studies have evaluated the correlation between CVRF and AAA. In contrast, only few studies focused on the association between subclinical ATS and CVRF with abdominal aortic diameter (AAD) and enlarged $A A D \geq 25 \mathrm{~mm}$ (EAAD) which has been demonstrated to be related to AAA risk [12]. To better elucidate AAA pathogenesis, the purpose of this study was to evaluate the influence of traditional CVRF and subclinical ATS on early stages of AAD enlargement among a population of adult patients at high risk of cardiovascular diseases (CVD). 


\section{Methods}

Study population and cardiovascular risk factors

Consecutive 2,052 patients, aged 20-80 years, referred to the Lipid Unit of a University Hospital for cardiovascular risk assessment (Framingham coronary heart disease RiskScore), and/or therapeutic advice, were enrolled. All participants gave informed written consent, and study protocol was approved by the local ethics committee. CVRF were prospectively collected as indicated here: patients answered a detailed questionnaire collecting information on family history of premature CHD, personal history of CVD, diabetes, hypertension, blood lipids, and current medications. Blood pressure was measured with a digital blood pressure monitor. Body mass index (BMI) and the Framingham coronary heart disease risk score were calculated for all patients.

All patients underwent venous blood drawing following an overnight fast. Total cholesterol, HDL cholesterol (HDL-C), triglycerides, and glucose were determined on fresh plasma samples using an auto-analyser (Cobras-Mira Plus $^{\mathrm{TM}}$, Roche, Basel, Switzerland).

Hypertension was defined as SBP $>140 \mathrm{mmHg}$ and/or DBP $>90 \mathrm{mmHg}$, and/or current use of anti-hypertensive agents. Smoking status was defined as current, former, or never. Obesity was defined as BMI $\geq 30 \mathrm{~kg} / \mathrm{m}^{2}$. Hypercholesterolemia was defined as plasma total cholesterol $>6.5 \mathrm{mmol} / \mathrm{L}$ and/or current use of lipid lowering therapy. Hypertriglyceridemia was defined as plasma triglyceride level $\geq 1.7 \mathrm{mmol} / \mathrm{L}$. Low HDL-C was defined as an HDL-C $<1 \mathrm{mmol} / \mathrm{L}$. Diabetes was defined by the current use of physician-prescribed antiglycemic medications or a fasting serum glucose level $\geq 7 \mathrm{mmol} / \mathrm{L}$. CHD was defined as a history of angina, acute coronary syndrome, or myocardial infarction. Cerebrovascular disease was defined as any history of transient ischemic attack or stroke. A positive family history of premature (men aged $<55$, and women $<65$ years) CHD was considered when there was a history of CHD in a first degree relative.

\section{Abdominal aorta evaluation}

B-mode ultrasound (US) imaging, using a $3.5-\mathrm{MHz}$ realtime sector scanner, was used to measure AAD below renal arteries. Abdominal aorta (AA) was examined both in antero-posterior and lateral views. Maximum external diameter within the infrarenal segment was measured. AA was considered enlarged if diameter was $\geq 25 \mathrm{~mm}$ but $<30 \mathrm{~mm}$, presence of AAA was defined as an AAD $\geq 30 \mathrm{~mm}[13]$.
Subclinical atherosclerosis evaluation

Carotid and femoral arteries were analyzed by B-mode US imaging. The analysis was performed with a colour duplex scanner, using a high resolution $7 \mathrm{MHz}$ linear array scan head. The duplex scan was coupled with the M'ATHS software performing semi-automatic on frame measures (Metris, Paris, France). Carotid arteries were examined from the lowest visible part, in the supraclavicular fossa, to the submandibular angle. Femoral arteries were scanned from $4 \mathrm{~cm}$ above, and $4 \mathrm{~cm}$ below, the inguinal bifurcation. Plaque was defined as a focal encroaching of $\geq 1.2 \mathrm{~mm}$ into the arterial lumen measured from mediaadventitia to intima-lumen interface. Plaque thickness was measured in its largest portion. Whenever more than one plaque were present within the same arterial segment, only the thickest one, measured in the longitudinal view, was recorded. To quantify ATS burden an "Atherosclerosis Burden Score" (ABS) ranging from 0 to 4 , reflecting the number of arterial bifurcations with plaques, was used.

\section{Statistical analyses}

All statistical analyses were performed using STATA 11.0 software (Stata corp., College station, TX, USA). The results were expressed as mean \pm standard deviation (SD), categorical data as percentages, or interquartile ranges. Comparison between categories was based on the $t$ test for continuous variables, and $\chi^{2}$ test for categorical variables. Association between aortic diameter, CVRF, and ABS were assessed with univariate (Pearson's and Spearman's rank) correlation, and multivariable linear modeling regression analysis. Finally, interaction between EAAD, CVRF, and other potential determinants, was assessed with logistic multivariate regression analysis.

\section{Results}

Consecutive 2,052 patients were included in the study. At the time of abdominal ultrasound, mean participants age was $52 \pm 13$ years (range from 20 to 80 years old). Sixty percent $(1,245)$ of included patients were men. General characteristics of studied population are summarized in Table 1. There were significant statistical differences between men and women concerning CVRF distribution. Hypertension, current and past smokers, hypertriglyceridemia, low HDL-C, personal history of CVD $(p<0.001)$, and diabetes $(p<0.01)$ were more frequent in men than in women. In contrast, there were more women with hypercholesterolemia and familial history of CHD than men $(p<0.001)$. The presence of subclinical ATS (ABS $\geq 1)$ was high in our study population (71\%). 
Table 1 Clinical characteristics of patients

Statistical differences: women versus men ${ }^{\dagger} p<0.01$, ${ }^{*} p<0.001$

Fig. 1 Distribution of abdominal aortic diameter by gender

\begin{tabular}{llll}
\hline Parameters & Women & Men & All \\
\hline Number (\%) & $807(39)$ & $1,245(61)$ & 2,052 \\
Age (mean \pm SD) & $53.2 \pm 14$ & $51.5 \pm 12^{\dagger}$ & $52.2 \pm 12.9$ \\
Familial history of premature CVD (\%) & 23.8 & $18.4^{\dagger}$ & 20.5 \\
Personal history of CVD (\%) & 4.3 & $10.9^{*}$ & 8.3 \\
Current smokers (\%) & 21.3 & $28.1^{*}$ & 25.4 \\
Past smokers (\%) & 15.9 & $29.6^{\ddagger}$ & 24.2 \\
Hypertension (\%) & 39 & $47.1^{*}$ & 43.9 \\
Hypercholesterolemia (\%) & 57.9 & $51.7^{\dagger}$ & 54.1 \\
Hypertriglyceridemia (\%) & 22.5 & $40.2^{*}$ & 33.2 \\
Low HDL cholesterolemia (\%) & 9.4 & $26.3^{*}$ & 19.6 \\
Obesity (\%) & 13.4 & 15.3 & 14.4 \\
Diabetes mellitus (\%) & 7.2 & $11.2^{\dagger}$ & 9.7 \\
Number of patients with ABS $\geq 1 \%)$ & 64.6 & $74.5^{*}$ & 70.6
\end{tabular}

Mean AAD increased with age ranging from $12.3 \pm$ $2.3 \mathrm{~mm}$, in 20-29 years old men, to $15.3 \pm 2.9 \mathrm{~mm}$ among those 70-80 years old. Among women, AAD increased from $14.0 \pm 1.7 \mathrm{~mm}$, in the 20-29 years old, to $18.4 \pm$ $4.5 \mathrm{~mm}$ among those aged 70-80 years. Thus, men showed significantly larger AAD than women (mean AAD of $16.4 \pm 2.9 \mathrm{~mm}$ vs. $14.2 \pm 1.9 \mathrm{~mm}$, respectively; $p<0.001)$ (Fig. 1).

Prevalence of patients with either an EAAD $\geq 20 \mathrm{~mm}$, $\geq 25 \mathrm{~mm}$, or AAA was respectively 4.0, 0.97, and $0.44 \%$. Among subjects $>65$ years old, this prevalence was respectively $3.9,1.1$, and $0.0 \%$ among women and 17.2, 5.2, and $2.3 \%$ among men.

As illustrated in Fig. 2, all parameters significantly correlated with AAD. However, this figure indicates that patients with EAAD are unequally far from regression line. When performing analysis using the univariate linear regression (Spearman's correlation) a significant correlation was observed between AAD and 12 of the 14 studied parameters. Vice versa, when multivariate analysis was performed, a correlation was found only for 6 of the parameters $\left(R^{2}=0.27, p<0.001\right)$ (Table 2$)$. However, this correlation was no more significant for AAD $\geq 20 \mathrm{~mm}$ $\left(R^{2}=0.14, p<0.54\right)$.

Probability to observe an EAAD $\geq 25 \mathrm{~mm}$ in association with all these same parameters was also analyzed (Fig. 3). Only gender, hypertriglyceridemia, current smoking, ABS, and age were found to be significantly associated with EAAD. However, these factors accounted for no more than $30 \%$ of interaction $\left(R^{2}=0.30, p<0.001\right)$.

\section{Discussion}

The results presented herein indicate that ATS burden, and some CVRF are positively correlated with AAD increase. However, in our population of middle-aged patients with increased cardiovascular risk, a subgroup of patients with an EAAD $\geq 20$ or $25 \mathrm{~mm}$ are unequally far from the regression line regardless of CVRF and CHD risk score. 

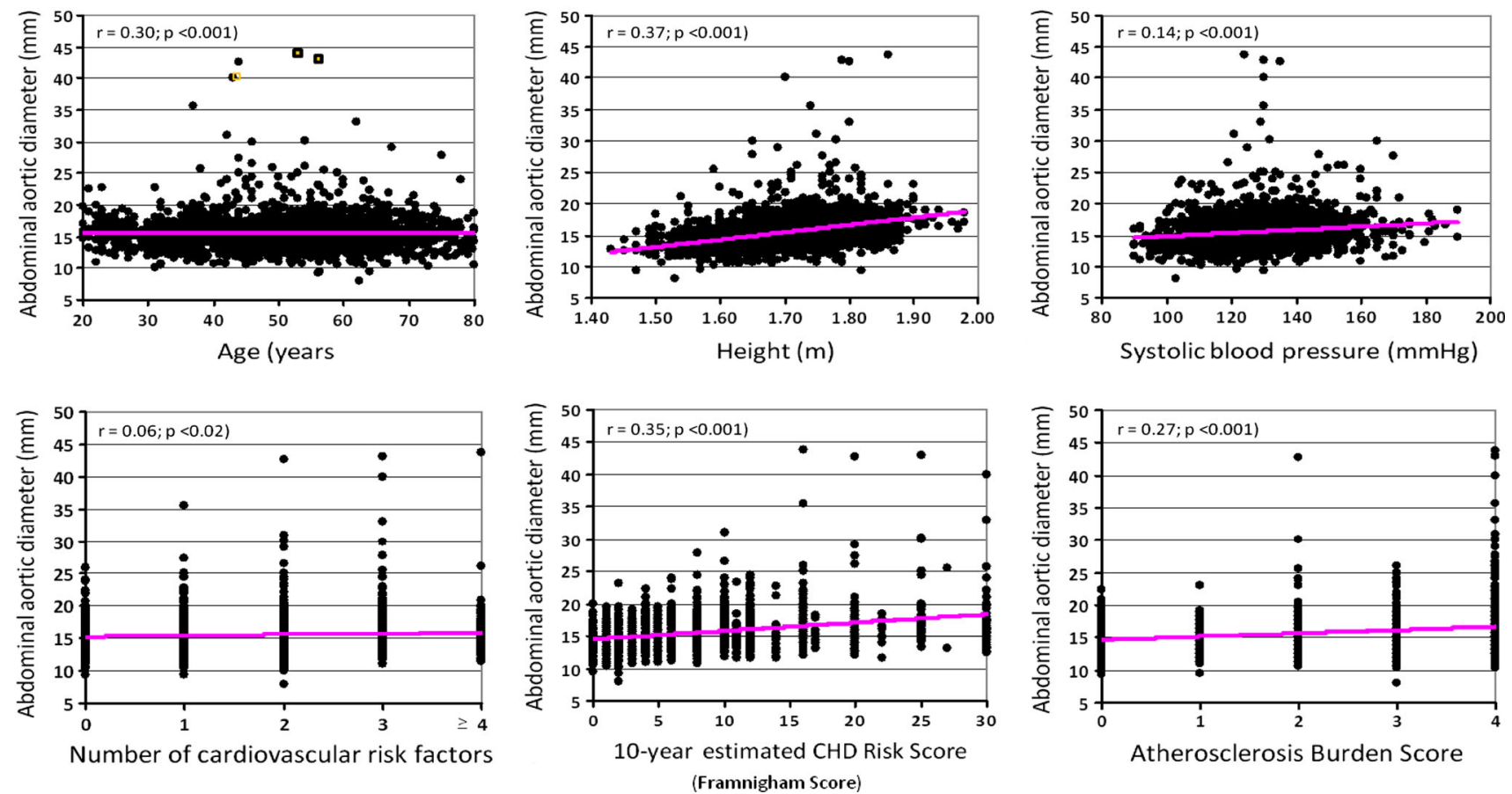

Fig. 2 Correlation (Pearson's) between the aortic abdominal diameter and cardiovascular risk factors and subclinical atherosclerosis

Table 2 Correlation between abdominal aortic diameter and traditional cardiovascular risk factors by univariate and multivariate regression analysis

\begin{tabular}{|c|c|c|c|c|}
\hline Variables & $\begin{array}{l}\text { Univariate regression analysis } \\
\text { Coefficient } \rho\end{array}$ & $\begin{array}{l}\text { Multivariate analysis } \\
p\end{array}$ & Coefficient $\beta$ & $p$ \\
\hline Gender (M vs. F) & 0.474 & 0.000 & 0.371 & 0.000 \\
\hline Age (decades) & 0.273 & 0.000 & 0.299 & 0.000 \\
\hline Familial history of premature CVD (yes vs. no) & 0.058 & 0.009 & -0.252 & 0.175 \\
\hline Personal history of CVD (yes vs. no) & -0.086 & 0.000 & 0.009 & 0.738 \\
\hline Atherosclerosis burden score ( $0-4$ units) & 0.257 & 0.000 & 0.049 & 0.039 \\
\hline Current smoking (yes vs. no) & -0.001 & 0.984 & 0.042 & 0.054 \\
\hline Past smoking (yes vs. no) & 0.147 & 0.000 & 0.038 & 0.083 \\
\hline Diabetes mellitus (yes vs. no) & 0.034 & 0.127 & -0.028 & 0.183 \\
\hline Systolic blood pressure (mmHg) & 0.148 & 0.000 & -0.750 & 0.004 \\
\hline Diastolic blood pressure $(\mathrm{mmHg})$ & 0.0145 & 0.000 & 0.081 & 0.009 \\
\hline Total cholesterol (mmol/L) & -0.134 & 0.000 & -0.006 & 0.801 \\
\hline HDL-cholesterol (mmol/L) & -0.168 & 0.000 & -0.012 & 0.561 \\
\hline Triglycerides (mmol/L) & 0.118 & 0.000 & 0.003 & 0.877 \\
\hline BMI $\left(\mathrm{kg} / \mathrm{m}^{2}\right)$ & 0.259 & 0.000 & 0.078 & 0.000 \\
\hline
\end{tabular}

$\rho$ Spearman's rank correlation coefficient, $\beta$ standardized regression coefficient

Few studies have been published assessing AAD epidemiology and particularly concerning the role of traditional CVRF on aortic diameter variability and early stages of AAA [14-18].

The present study is in agreement with the previous ones showing a link between AAD enlargement with aging and male gender $[19,20]$. With age, arteries undergo changes in their structural and functional capacities. Arterial wall collagen-to-elastin ratio increases, as a result of decreased elastic elements, leading to arterial dilation. In parallel, arterial wall stiffness tends to increase [19]. Gender differences in AAD are probably explained by higher height and stiffness in AA of men [21, 22].

When concerning the other CVRF, a positive correlation between BMI and AAD increment was observed in our patients similarly to what reported by others [14-16]. This 
Fig. 3 Interaction between enlarged abdominal aortic diameter $(\geq 25 \mathrm{~mm})$ and cardiovascular risk factors and subclinical atherosclerosis, by multivariate regression analysis

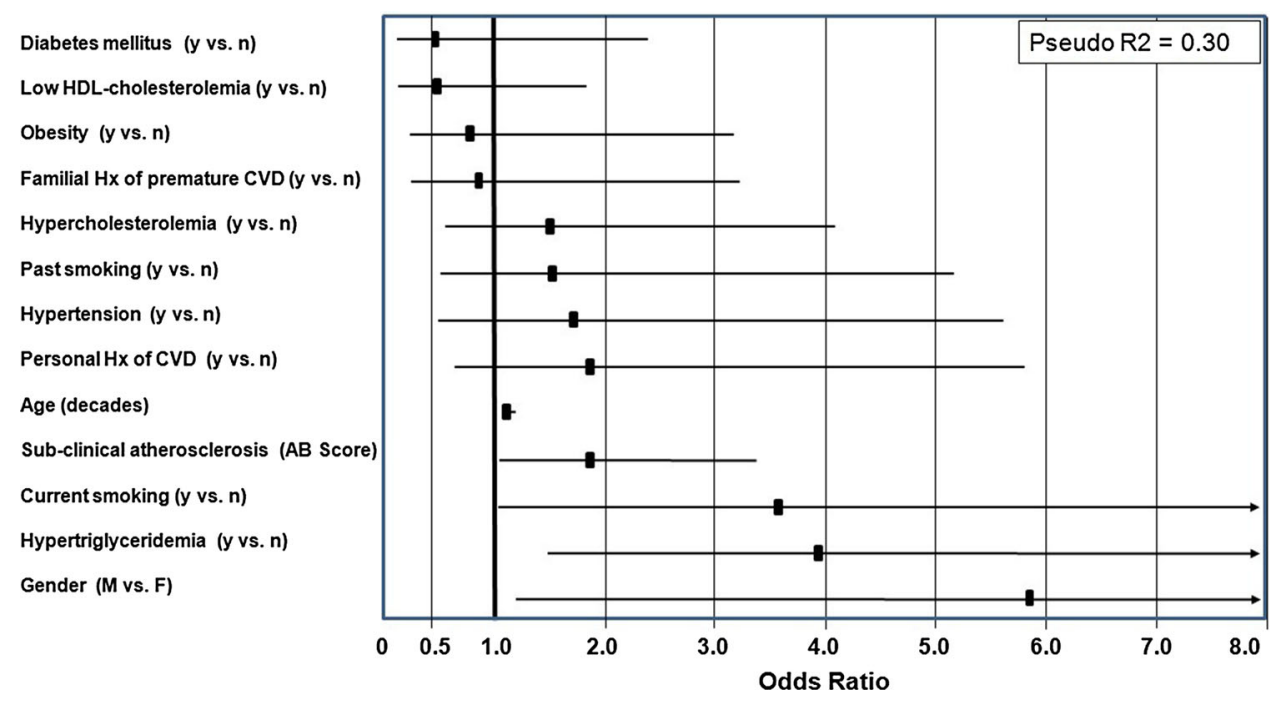

association may be explained by the ability of adipose tissue to release adipokines. Indeed, it has been shown that adipokines, including resistin, adiponectin, and leptin, can play a paramount role in AAA pathogenesis [4].

Hypertension has limited effect on arterial diameter. In contrast to Paivansalo et al. [16], we and others observed a correlation between hypertension and weak AAD increment $[14,18,19]$. Increase in arteries diameter has been advocated as a possible increase of fluid volume secondary to sodium retention [23].

Although smoking constitutes a significant risk for aneurysm development, two previous studies [14, 15], evaluating impact of CVRF on AAD, have shown lack of association between smoking and AAD. Paivansalo et al. [16]. describe a significant correlation in smoking women only $(p<0.005)$. In our study, correlation was nearly significant $(p=0.054)$. More recently, Johnsen et al. [17] have shown that AAD increases markedly, between 18 and $29 \mathrm{~mm}$, with current smoking ( $p<0.0001)$. Finally, in the MESA study [18], the current smoking was independently and significantly associated with increasing AAD $(p<0.01)$.

Lastly, we did not find any correlation between AAD and diabetes. This result is in accordance with the previous studies demonstrating inverse or no association between AAD and diabetes $[8,14]$.

Statistical multivariate regression analysis revealed a significant association between subclinical ATS burden score (ABS) and AAD increment. This result is in agreement with others demonstrating similar association using different tools or techniques to quantify subclinical ATS burden. Two studies [14, 18], demonstrated a significant association between increasing $\mathrm{AAD}$ and presence and extent of calcified ATS in AA and iliac arteries, or coronary calcium using EBCT and calcium score. Patel et al.
[15] found similar association using ultrasounds to measure $\mathrm{AAD}$ and IMT at several AA locations. AAD increase in the presence of ATS may be explained by arterial remodelling: preservation of arterial lumen cross-sectional area in spite of advancing ATS within the arterial wall (Glagov's phenomenon) [24]. Labropoulus et al. studied vessel diameters throughout the vascular system in $67 \pm 12$-yearold subjects with and without ATS and found that all arteries dilate during early ATS plaque formation confirming the Glagov's phenomenon throughout the vascular tree [25]. Finally, Mohiaddin et al. [26] confirmed the presence of Glagov remodeling in AA by cardiovascular magnetic resonance, with an increment of $1 \mathrm{~mm}$ over 2 years in patients with arterial plaques.

An association between some CVRF and AAD does not necessarily imply a link of causality. Multivariate regression analysis showed that CVRF and ABS accounted for only $27 \%\left(R^{2}=0.27\right)$ of AAD variability in our study.

When concerning the subgroup of 20 patients with EAAD $\geq 25 \mathrm{~mm}$, only age, gender, current smoking, hypertriglyceridemia, and ABS appeared to be significant positive predictors. These risk factors are similar to those known for AAA [27, 28].

Interestingly, despite a large proportion of patients with diffuse ATS lesions ( $71 \%$ ) and $\geq 3$ CVRF (37\%), prevalence of EAAD and AAA was low in our study population, 0.97 and $0.44 \%$, respectively. Among men older than 65 years of age, the $5.2 \%$ EADD and the $2.3 \%$ AAA prevalence were lower than those found in other studies. Indeed, screening studies show that AAA occurs in 4-9\% of individuals over the age of $60[1,3]$.

If ATS was the dominant feature of aortic enlargement, the proportion of subjects with an aortic dilation should be widely superior. In our study, the dilation of aortic diameter was observed only in $2.4 \%$ of patients with an ABS 
score $\geq 3$, and in $2.9 \%$ of those with a history of coronary artery disease. In the subgroup of patients with $\mathrm{AAD} \geq 20 \mathrm{~mm}(n=83)$, these rates were 9.3 and $12.9 \%$, respectively. Moreover, the subpopulation of patients with AAD $\geq 25 \mathrm{~mm}$ was scattered regardless of CVRF number, CHD risk score, or ATS burden. Herein, these traditional CVRF accounted for a role in AAD enlargement for no more than $30 \%$ as expressed by the pseudo- $R^{2}$. Similarly, in the Troms $\varnothing$ Study, no consistent dose-response relationship between ATS and AAD was found [18]. These observations strongly suggest that other factors than CVRF and ATS are implicated in EAAD and ultimately AAA formation. Thus, ATS may not be a causal but rather a predisposing contributor for AAA. Indeed, recent studies demonstrated the paramount role played by inflammation, proteolysis, apoptosis, and oxidative stress in AAA development and progression underlying the systemic nature of the aneurysmal process $[8,29,30]$.

The cross-sectional nature of the present study and the lack of direct atheroma measurement within the aorta have to be considered as limitations that possibly interfere on the causal relationship between ATS, CVRF, and AAD increment. Nevertheless, this study provides additional evidence suggesting that pathophysiology of EAAD and AAA is complex and multifactorial.

Our results demonstrate that traditional CVRF and ATS only modestly contribute to AAD increase and are poor predictors of EAAD, supporting the view that other key factors need to be searched to further improve knowledge of pathophysiology and AAA prediction.

Acknowledgments The authors wish to thank Alice Jaltier for her precious technical assistance.

Conflict of interest None declared.

\section{References}

1. Lederle FA, Johnson GR, Wilson SE, Chute EP, Littooy FN, Bandyk D, Krupski WC, Barone GW, Acher CW, Ballard DJ (1997) Prevalence and associations of abdominal aortic aneurysm detected through screening. Aneurysm Detection and Management (ADAM) Veterans Affairs Cooperative Study Group. Ann Int Med 126(6):441-449

2. Reed D, Reed C, Stemmermann G, Hayashi T (1992) Are aortic aneurysms caused by atherosclerosis? Circulation 85(1):205-211

3. Scott RA, Ashton HA, Kay DN (1991) Abdominal aortic aneurysm in 4237 screened patients: prevalence, development and management over 6 years. Br J Surg 78(9):1122-1125

4. Golledge J, Clancy P, Jamrozik K, Norman PE (2007) Obesity, adipokines, and abdominal aortic aneurysm: health in Men study. Circulation 116(20):2275-2279

5. Iribarren C, Darbinian JA, Go AS, Fireman BH, Lee CD, Grey DP (2007) Traditional and novel risk factors for clinically diagnosed abdominal aortic aneurysm: the Kaiser multiphasic health checkup cohort study. Ann Epidemiol 17(9):669-678
6. Hobbs SD, Claridge MW, Quick CR, Day NE, Bradbury AW, Wilmink AB (2003) LDL cholesterol is associated with small abdominal aortic aneurysms. Eur J Vasc Endovasc Surg 26(6): 618-622

7. Norrgard O, Angquist KA, Johnson O (1985) Familial aortic aneurysms: serum concentrations of triglyceride, cholesterol, HDL-cholesterol and (VLDL + LDL)-cholesterol. Br J Surg 72(2):113-116

8. Golledge J, Muller J, Daugherty A, Norman P (2006) Abdominal aortic aneurysm: pathogenesis and implications for management. Arterioscler Thromb Vasc Biol 26(12):2605-2613

9. Klink A, Hyafil F, Rudd J, Faries P, Fuster V, Mallat Z, Meilhac O, Mulder WJ, Michel JB, Ramirez F, Storm G, Thompson R, Turnbull IC, Egido J, Martin-Ventura JL, Zaragoza C, Letourneur D, Fayad ZA (2011) Diagnostic and therapeutic strategies for small abdominal aortic aneurysms. Nat Rev Cardiol 8(6):338-347

10. Cabellon S Jr, Moncrief CL, Pierre DR, Cavanaugh DG (1983) Incidence of abdominal aortic aneurysms in patients with atheromatous arterial disease. Am J Surg 146(5):575-576

11. MacSweeney ST, O’Meara M, Alexander C, O'Malley MK, Powell JT, Greenhalgh RM (1993) High prevalence of unsuspected abdominal aortic aneurysm in patients with confirmed symptomatic peripheral or cerebral arterial disease. Br J Surg 80(5):582-584

12. Solberg S, Forsdahl SH, Singh K, Jacobsen BK (2010) Diameter of the infrarenal aorta as a risk factor for abdominal aortic aneurysm: the Tromso Study, 1994-2001. Eur J Vasc Endovasc Surg 39(3):280-284

13. Hirsch AT, Haskal ZJ, Hertzer NR, Bakal CW, Creager MA, Halperin JL, Hiratzka LF, Murphy WR, Olin JW, Puschett JB, Rosenfield KA, Sacks D, Stanley JC, Taylor LM Jr, White CJ, White J, White RA, Antman EM, Smith SC Jr, Adams CD, Anderson JL, Faxon DP, Fuster V, Gibbons RJ, Hunt SA, Jacobs AK, Nishimura R, Ornato JP, Page RL, Riegel B (2006) ACC/ AHA 2005 Practice Guidelines for the management of patients with peripheral arterial disease (lower extremity, renal, mesenteric, and abdominal aortic): a collaborative report from the American Association for Vascular Surgery/Society for Vascular Surgery, Society for Cardiovascular Angiography and Interventions, Society for Vascular Medicine and Biology, Society of Interventional Radiology, and the ACC/AHA Task Force on Practice Guidelines (Writing Committee to Develop Guidelines for the Management of Patients With Peripheral Arterial Disease): endorsed by the American Association of Cardiovascular and Pulmonary Rehabilitation; National Heart, Lung, and Blood Institute; Society for Vascular Nursing; TransAtlantic InterSociety Consensus; and Vascular Disease Foundation. Circulation 113(11):e463-e654

14. Allison MA, Kwan K, DiTomasso D, Wright CM, Criqui MH (2008) The epidemiology of abdominal aortic diameter. J Vasc Surg 48(1):121-127

15. Patel AS, Mackey RH, Wildman RP, Thompson T, Matthews K, Kuller L, Sutton-Tyrrell K (2005) Cardiovascular risk factors associated with enlarged diameter of the abdominal aortic and iliac arteries in healthy women. Atherosclerosis 178(2):311-317

16. Paivansalo MJ, Merikanto J, Jerkkola T, Savolainen MJ, Rantala AO, Kauma H, Lilja M, Reunanen YA, Kesaniemi A, Suramo I (2000) Effect of hypertension and risk factors on diameters of abdominal aorta and common iliac and femoral arteries in middle-aged hypertensive and control subjects: a cross-sectional systematic study with duplex ultrasound. Atherosclerosis 153(1): 99-106

17. Johnsen SH, Forsdahl SH, Singh K, Jacobsen BK (2010) Atherosclerosis in abdominal aortic aneurysms: a causal event or a process running in parallel? The Tromso Study. Arterioscler Thromb Vasc Biol 30(6):1263-1268 
18. Laughlin GA, Allison MA, Jensky NE, Aboyans V, Wong ND, Detrano R, Criqui MH (2011) Abdominal aortic diameter and vascular atherosclerosis: the Multi-Ethnic Study of Atherosclerosis. Eur J Vasc Endovasc Surg 41(4):481-487

19. Dixon AK, Lawrence JP, Mitchell JR (1984) Age-related changes in the abdominal aorta shown by computed tomography. Clin Radiol 35(1):33-37

20. Pearce WH, Slaughter MS, LeMaire S, Salyapongse AN, Feinglass J, McCarthy WJ, Yao JS (1993) Aortic diameter as a function of age, gender, and body surface area. Surgery 114(4):691-697

21. Lanne T, Sonesson B, Bergqvist D, Bengtsson H, Gustafsson D (1992) Diameter and compliance in the male human abdominal aorta: influence of age and aortic aneurysm. Eur J Vasc Surg 6(2):178-184

22. Lakatta EG, Levy D (2003) Arterial and cardiac aging: major shareholders in cardiovascular disease enterprises: part I: aging arteries: a "set up" for vascular disease. Circulation 107(1):139-146

23. Ward MR, Pasterkamp G, Yeung AC, Borst C (2000) Arterial remodeling: mechanisms and clinical implications. Circulation 102(10):1186-1191

24. Glagov S, Weisenberg E, Zarins CK, Stankunavicius R, Kolettis GJ (1987) Compensatory enlargement of human atherosclerotic coronary arteries. New Engl J Med 316(22):1371-1375

25. Labropoulos N, Zarge J, Mansour MA, Kang SS, Baker WH (1998) Compensatory arterial enlargement is a common pathobiologic response in early atherosclerosis. Am J Surg 176(2):140-143

26. Mohiaddin RH, Burman ED, Prasad SK, Varghese A, Tan RS, Collins SA, Hughes RL, Gatehouse PD, Jhooti P, Longmore DB, Yang GZ, Firmin DN, Pennell DJ (2004) Glagov remodeling of the atherosclerotic aorta demonstrated by cardiovascular magnetic resonance: the CORDA asymptomatic subject plaque assessment research (CASPAR) project. J Cardiovasc Magn Reson 6(2):517-525

27. Forsdahl SH, Singh K, Solberg S, Jacobsen BK (2009) Risk factors for abdominal aortic aneurysms: a 7-year prospective study: the Tromso Study, 1994-2001. Circulation 119(16):22022208

28. Aggarwal S, Qamar A, Sharma V, Sharma A (2011) Abdominal aortic aneurysm: a comprehensive review. Exp Clin Cardiol 16(1):11-15

29. Furubayashi K, Takai S, Jin D, Miyazaki M, Katsumata T, Inagaki S, Kimura M, Tanaka K, Nishimoto M, Fukumoto H (2008) Chymase activates promatrix metalloproteinase- 9 in human abdominal aortic aneurysm. Clin Chim Acta 388(1-2):214-216

30. Nordon IM, Hinchliffe RJ, Malkawi AH, Pirianov G, Torsney E, Loftus IM, Cockerill GW, Thompson MM (2011) Comparative proteomics reveals a systemic vulnerability in the vasculature of patients with abdominal aortic aneurysms. J Vasc Surg 54(4): 1100 e6-1108 e6 